\title{
AMENABLE PAIRS OF GROUPS AND ERGODIC ACTIONS AND THE ASSOCIATED VON NEUMANN ALGEBRAS ${ }^{1}$ \\ BY
}

ROBERT J. ZIMMER

\begin{abstract}
If $X$ and $Y$ are ergodic $G$-spaces, where $G$ is a locally compact group, and $X$ is an extension of $Y$, we study a notion of amenability for the pair $(X, Y)$. This simultaneously generalizes and expands upon previous work of the author concerning the notion of amenability in ergodic theory based upon fixed point properties of affine cocycles, and the work of Eymard on the conditional fixed point property for groups. We study the relations between this concept of amenability, properties of the von Neumann algebras associated to the actions by the Murray-von Neumann construction, and the existence of relatively invariant measures and conditional invariant means.
\end{abstract}

1. Introduction. If $G$ is a locally compact second countable group, the notion of amenability for $G$ can be characterized in a variety of ways. Two of the most important are the fixed point property for affine actions and the existence of an invariant mean on $L^{\infty}(G)$. For $G$ discrete, amenability can also be characterized in terms of the von Neumann algebra generated by the regular representation. If $H$ is a subgroup of $G$, the notion of amenability for the pair $(H, G)$ was examined in detail by Eymard [4], who showed, among other things, that the existence of an invariant mean on $L^{\infty}(G / H)$ is equivalent to a conditional fixed point property.

In [14], the author introduced the notion of amenability for an ergodic action of a locally compact group, which plays a role in ergodic theory parallel to that of amenability in group theory. Amenability for ergodic group actions is based upon an analogue of the fixed point property for groups, namely an "invariant section property" for affine cocycles. When the group is countable and discrete, amenable actions may be characterized in terms of an analogue of a $G$-invariant mean, and also in terms of the von Neumann algebra associated to the action by the classical Murray-von Neumann group measure space construction [15], [16]. The point of this paper, which may be considered a sequel to [14], [15], and [16], is to develop the analogue of Eymard's notion in ergodic theory; namely, we consider amenable pairs of ergodic $G$-spaces $(X, Y)$ where $X$ is a $G$-space extension of $Y$. Our definition

Received by the editors June 8, 1977.

AMS (MOS) subject classifications (1970). Primary 22D40, 28A65, 46L10; Secondary 22D25.

${ }^{1}$ Research supported by NSF Grant MCS76-00626. 
of amenability for the pair is in terms of a conditional invariant section property. (For the case in which $Y$ is a point, the author also considered this notion in [14].) In $\$ 2$ below, we present the definition and some basic properties. For $G$ countable and discrete, we show in $\$ 4$ that amenability of the pair $(X, Y)$ is equivalent to the existence of a $G$-invariant conditional mean $L^{\infty}(X) \rightarrow L^{\infty}(Y)$, and can be characterized as well in terms of the existence of a norm one projection between the associated von Neumann algebras. $\S 3$ is concerned with questions of existence of extensions of a given action which form an amenable pair. We show that every ergodic action of $G$ on $Y$ has an ergodic extension $X$ such that $(X, Y)$ is an amenable pair, and, moreover, that every ergodic system of imprimitivity based on $Y$ arises as a subsystem of a system of imprimitivity naturally induced by some such extension of $Y$. These extensions may in fact be chosen to have a relatively invariant measure, a condition stronger than amenability of the pair. The results of $\$ 3$ are based on I. Segal's work concerning the canonical normal distribution on a Hilbert space.

2. Preliminaries. We begin by recalling the definitions of amenable ergodic actions and amenable pairs introduced in [14]. We refer the reader to that paper for a discussion of the definitions to follow. Let $G$ be a locally compact second countable group. By an ergodic $G$-space we will mean a standard Borel space $S$ with a Borel right $G$-action, $S \times G \rightarrow S$, and a probability measure $\mu$, quasi-invariant and ergodic under $G$. If $M$ is a standard Borel group, a Borel function $\alpha: S \times G \rightarrow M$ is called a cocycle if for all $g, h \in G$, $\alpha(s, g h)=\alpha(s, g) \alpha(s g, h)$ for almost all $s$. If $\pi: G \rightarrow M$ is a homomorphism, then $\alpha(s, g)=\pi(g)$ defines a cocycle called the restriction of $\pi$ to $S \times G$. Let $E$ be a separable Banach space, $E^{*}$ the dual, and $E_{1}^{*}$ the unit ball in the dual, which is compact and metrizable in the $\sigma\left(E^{*}, E\right)$ topology. Let Iso $(E)$ be the group of isometric isomorphisms of $E$, which is a standard Borel group in the strong operator topology [14, Lemma 1.1]. If $S$ is a standard Borel space, by a Borel field of compact convex subsets of $E_{1}^{*}$ we mean an assignment $s \rightarrow A_{s}$, where $A_{s} \subset E_{1}^{*}$ is compact and convex, and $\tilde{A}=\left\{(s, x) \mid x \in A_{s}\right\} \subset S \times E_{1}^{*}$ 1s Borel. Suppose that $\alpha: S \times G \rightarrow \operatorname{Iso}(E)$ is a cocycle. Then there is an induced adjoint cocycle $\alpha^{*}: S \times G \rightarrow \operatorname{Homeo}\left(E_{1}^{*}\right)$ defined by $\alpha^{*}(s, g)=$ $\left(\alpha(s, g)^{-1}\right)^{*}$. A Borel field is called $\alpha$-invariant if for each $g, \alpha^{*}(s, g) A_{s g}=A_{s}$ a.e. A Borel function $\varphi: S \rightarrow E_{1}^{*}$ will be called an $\alpha$-invariant section if for all $g, \alpha^{*}(s, g) \varphi(s g)=\varphi(s)$ a.e. We call $S$ an amenable $G$-space if every $\alpha$-invariant field of compact convex sets contains an $\alpha$-invariant section. More precisely:

DEFINITION 2.1. If $S$ is an ergodic $G$-space, we call $S$ amenable if for every separable Banach space $E$, cocycle $\alpha: S \times G \rightarrow \operatorname{Iso}(E)$, and $\alpha$-invariant field 
$A_{s}$, there exists an $\alpha$-invariant section with $\varphi(s) \in A_{s}$ a.e. (We call $\varphi$ a section in $A_{s}$ )

Thus amenability of $S$ is a type of fixed point (or "invariant section") property. In fact, if $S$ is a transitive $G$-space, then this condition is equivalent to the usual fixed point property for the stability groups [14, Theorem 1.9]. In [14], we also introduced the notion of an amenable pair $(S, G)$, where $S$ is an ergodic $G$-space, generalizing the notion of a conditional fixed point property introduced by Eymard.

Definition 2.2. If $S$ is an ergodic $G$-space, $(S, G)$ is called an amenable pair if for every (continuous) homomorphism $\pi: G \rightarrow \operatorname{Iso}(E)$, and $G$-invariant compact convex set $A \subset E_{1}^{*}$, the existence of an $\alpha$-invariant section $\varphi: S \rightarrow A$ (where $\alpha$ is the restriction of $\pi$ to $S \times G$ ) implies the existence of a fixed point for $G$ in $A$.

Proposition 2.3 [14, Proposition 4.2]. If $S=G / H$ is a transitive G-space, then $(S, G)$ is an amenable pair if and only if the pair $(H, G)$ has the conditional fixed point property of Eymard [4, p. 11].

The following result was proved in [14].

Proposition 2.4 [14, Proposition 4.4]. If $S$ is an ergodic G-space and there is a G-invariant mean on $L^{\infty}(S)$, then $(S, G)$ is an amenable pair.

If $S$ is a transitive $G$-space, it follows from Proposition 2.3 and a result of Eymard that the converse of this proposition is true. We shall see in $\$ 4$ that at least for $G$ countable and discrete the converse is true for any ergodic $S$.

In [14], we indicated that one could define amenability of a pair $(X, Y)$ where $X$ is an extension of $Y$ by a conditional invariant section property, and we present this definition now. Recall that if $(X, \mu),(Y, \nu)$ are ergodic $G$-spaces, then $X$ is called an extension of $Y$ if there exists a $G$-invariant Borel map $p: X \rightarrow Y$ such that $p_{*}(\mu)=\nu$. (Actually, $p$ need only be defined on a $G$-invariant conull Borel subset of $X$, but as this technicality will play no role in what follows, we shall ignore it.) If $\beta: Y \times G \rightarrow M$ is a cocycle, then the restriction of $\beta$ to $X \times G$, say $\alpha$, is defined by $\alpha(x, g)=\beta(p(x), g)$. If $Y$ is a point, then $\beta$ is just a homomorphism defined on $G$, and the restriction of $\beta$ to $X \times G$ coincides with our previous definition.

Definition 2.5. If $p: X \rightarrow Y$ is an extension of $Y$, then $(X, Y)$ is an amenable pair if for every cocycle $\beta: Y \times G \rightarrow \operatorname{Iso}(E)$ and $\beta$-invariant Borel field of compact convex sets $A_{y} \subset E_{1}^{*}$, the existence of an $\alpha$-invariant section in $A_{x}$ (where $A_{x}$ is defined by $A_{x}=A_{p(x)}$ ) implies the existence of a $\beta$ invariant section in $A_{y}$.

We remark that if $Y$ is an amenable $G$-space, then $(X, Y)$ is an amenable pair for every extension $X$ of $Y$. We now discuss some other natural 
onditions on an extension which implies amenability of the pair $(X, Y)$.

If $p:(X, \mu) \rightarrow(Y, \nu)$ is an extension of ergodic actions, let $\mu=\int^{\oplus} \mu_{y} d \nu$ be a decomposition of $\mu$ over the fibers of $p$. For $g \in G$, we have that for almost all $y, \mu_{y} \cdot g$ and $\mu_{y g}$ are equivalent measures.

Defintion 2.6. We will call $\mu$ relatively invariant over $Y$ if for each $g$, $\mu_{y} \cdot g=\mu_{y g}$ for almost all $y$.

We remark that a similar notion in a somewhat different context has been considered by Glasner [5]. If $Y$ is a point, then a relatively invariant measure over $Y$ is just the usual notion of a $G$-invariant measure.

Proposition 2.7. If $X$ has a relatively invariant measure over $Y$, then $(X, Y)$ is an amenable pair.

Proof. With notation as above, let $\varphi$ be an $\alpha$-invariant section, $\varphi(x) \in$ $A_{p(x)}$ a.e. Define $\psi(y)=\int \varphi(x) d \mu_{y}$. Then $\psi$ is a Borel map, $\psi(y) \in A_{y}$, and

$$
\begin{aligned}
\psi(y g) & =\int \varphi(x) d \mu_{y g}(x) \\
& =\int \varphi(x) d\left(\mu_{y} \cdot g\right)(x)=\int \varphi(x g) d \mu_{y}(x) \text { a.e. } \\
& =\int \alpha^{*}(p(x), g)^{-1} \varphi(x) d \mu_{y}(x)=\beta^{*}(y, g)^{-1} \int \varphi(x) d \mu_{y}(x) \\
& =\beta^{*}(y, g)^{-1} \psi(y) .
\end{aligned}
$$

Thus, $\psi$ is a $\beta$-invariant section in $A_{y}$.

A more general notion than a relatively invariant measure is a generalization of the notion of an invariant mean.

Definition 2.8. If $X$ is an extension of $Y$, by an invariant conditional mean on $L^{\infty}(X)$ over $Y$ we mean a projection operator of norm one $M: L^{\infty}(X) \rightarrow$ $L^{\infty}(Y)$, with $M(1)=1$, and which is a $G$-map.

We note that it follows that $M$ must be positive, and satisfy $M(f h)=$ $f M(h)$ if $f \in L^{\infty}(Y)$ [12]. It is clear that if $\mu$ is a relatively invariant measure over $Y$, then $M(f)(y)=\int f d \mu$ defines an invariant conditional mean. If $Y$ is a point, then an invariant conditional mean is simply a $G$-invariant mean on $L^{\infty}(X)$. The following generalizes Proposition 2.4.

PROPOSITION 2.9. If there is an invariant conditional mean on $L^{\infty}(X)$ over $Y$, then $(X, Y)$ is an amenable pair.

Proof. With notation as above, let $\varphi$ be an $\alpha$-invariant section in $\left\{A_{p(x)}\right\}$. For each $w \in E$, define $f_{w} \in L^{\infty}(X)$ by $f_{w}(x)=\langle\varphi(x), w\rangle$, where $\langle$, $\rangle$ is the pairing $E^{*} \times E \rightarrow \mathrm{C}$. Then $w \rightarrow M\left(f_{w}\right)$ is a continuous map $E \rightarrow L^{\infty}(Y)$ of norm not exceeding one, and hence there is a weakly measurable $\psi: Y \rightarrow E_{1}^{*}$ such that for all $w, M\left(f_{w}\right)(y)=\langle\psi(y), w\rangle$ a.e. [2, p. 582]. Changing $\psi$ on a 
conull set if necessary, we can assume $\psi$ is Borel and is determined up to equality on a conull set by this equation. We now claim that for any essentially bounded and measurable map $\theta: Y \rightarrow E$,

$$
M(\langle\varphi(x), \theta(p(x))\rangle)(y)=\langle\psi(y), \theta(y)\rangle \text { a.e. }
$$

This can be shown exactly as in the proof of the lemma of [15], first showing this for simple $\theta$, and then using an approximation argument. With this in hand, we can now show that $\psi$ is actually a $\beta$-invariant section. We have

$$
\begin{aligned}
\left\langle\beta^{*}(y, g) \psi(y g), w\right\rangle & =\left\langle\psi(y g), \beta(y, g)^{-1} w\right\rangle \\
& =\left\langle\psi(y g), \beta\left(y g, g^{-1}\right) w\right\rangle \\
& =M\left(\left\langle\varphi(x), \beta\left(p(x), g^{-1}\right) w\right\rangle\right)(y g) \\
& =M\left(\left\langle\varphi(x g), \alpha\left(x g, g^{-1}\right) w\right\rangle\right)(y) \\
& =M\left(\left\langle\alpha^{*}(x, g) \varphi(x g), w\right\rangle\right)(y) \\
& =\langle\psi(y), w\rangle .
\end{aligned}
$$

Thus $\beta^{*}(y, g) \psi(y g)=\psi(y)$ a.e. Finally we must show that $\psi(y) \in A_{y}$ a.e. We argue as in the conclusion of [15]. Let $\theta$ run through a countable dense set in $E$ and $q$ be a rational number. It suffices to show $\theta\left(A_{y}\right)>q$ implies $\theta(\psi(y)) \geqslant q$ a.e. Let $Y_{0}=\left\{y \mid \theta\left(A_{y}\right) \geqslant q\right\}$. Then $Y_{0}$ is measurable. If $Y_{0}$ has positive measure, we have $\left.M\left(\langle\theta, \varphi(x)\rangle \chi_{p^{-1}\left(Y_{0}\right)}\right)\right\rangle M\left(q \chi_{p^{-1}\left(Y_{0}\right)}\right)=q \chi_{Y_{0}}$. Thus for almost all $y \in Y_{0},\langle\theta, \psi(y)\rangle \geqslant q$, completing the proof.

Just as with Proposition 2.4, we shall see in $\$ 4$ that the converse of Proposition 2.9 holds if $G$ is countable and discrete.

3. Existence questions. In this section we make some observations about the existence of extensions of a given action forming an amenable pair, and in fact about existence of extensions with relatively invariant measure. The simplest type of extension is just a product action, and for each $G$ we shall see that there is a $G$-space with finite invariant measure whose product with any $G$-space other than translation on $G$ modulo a compact subgroup is ergodic. The remaining case is easily handled, and we conclude that any ergodic $G$-space other than translation on $G$ itself has an ergodic extension with relatively invariant measure. For any extension $X \rightarrow Y$, the induced representation of $G$ on $L^{2}(X)$ defines an ergodic system of imprimitivity based on $Y$, and we also show, using similar techniques, that most ergodic systems of imprimitivity based on $Y$ appear as a subsystem of such a system, where $X$ has a relatively invariant measure over $Y$. These results are based on some properties of the canonical normal distribution on a Hilbert space, which we now recall. The reader is referred to [6, [10] for details. 
Let $H_{0}$ be a (separable) real Hilbert space with complexification $H$. Let $\mu$ be canonical normal distribution on $H_{0}$ (with parameter 1). Then $\mu$ is not $\sigma$-additive, but there is a standard Borel space $S$, a Borel probability measure $\nu$ on $S$, and a weak distribution $L: H_{0} \rightarrow F(S)$, where $F(S)$ is the space of measurable functions on $S$, so that any element of $H_{0}$, as well as any tame function on $H_{0}$, can be represented as a random variable on $S$. Let $\mathcal{P}$ be the algebra of tame polynomial functions on $H_{0}$, $C$ the algebra of bounded tame functions, and $\tilde{\mathcal{P}}$ and $\tilde{\mathcal{C}}$ the corresponding subalgebras of $F(S)$. Let $S(H)=$ $\sum_{k=0}^{\infty} S^{k}(H)$ be the symmetric tensor algebra of $H$, which is of course also a complex Hilbert space. If $U$ is a unitary on $H$, let $S(U)$ be the induced unitary on $S(H), S(U)=\Sigma^{\oplus} S^{k}(U)$. Then by [10, Corollary 3.1] there is a unitary isomorphism $D: S(H) \rightarrow L^{2}(S, \nu)$ such that for any $U$ which takes $H_{0} \subset H$ onto itself, we have for $f \in \mathcal{P}$ and the corresponding $\tilde{f} \in \tilde{\mathcal{P}}$, $D S(U) D^{-1} \tilde{f} \in \tilde{\mathcal{P}}$ corresponds to the element in $\mathscr{P}$ defined by $f \circ U^{*}$. Since every bounded measurable function on a finite dimensional Euclidean space is the $L^{2}$-limit (Gaussian measure) of polynomials, it follows by a limiting argument that the correspondence above for $f \in \mathscr{P}$ holds for $f \in \mathcal{C}$ as well. Now $f \rightarrow f \circ U^{*}$ is clearly multiplicative, so we can conclude that $D S(U) D^{-1}$ is multiplicative on $C$. Now $C \subset L^{\infty}(S)$ is an $L^{2}$-dense subalgebra and it follows by an approximation argument that $D S(U) D^{-1}\left(L^{\infty}(S)\right)=L^{\infty}(S)$, and that $D S(U) D^{-1}$ is an automorphism of this algebra.

THEOREM 3.1. For every noncompact locally compact second countable group, there is a standard Borel G-space, $S, a$ G-invariant probability measure $\nu$ on $S$, ergodic under $G$, such that the induced unitary representation of $G$ on $L^{2}(S) \ominus$ $C$ has the properties that (i) $U\left(g_{n}\right) \rightarrow 0$ weakly for every sequence $g_{n} \in G$ for which $g_{n} \rightarrow \infty$; and (ii) For every $f \in L^{2}(S), f \neq 1,\{g \mid U(g) f=f\}$ is a compact subgroup of $G$.

Proof. Take $H_{0}=L^{2}(G, \mathbf{R})$ in the above construction and let $\pi$ be the regular representation of $G$ on $H=L^{2}(G, \mathrm{C})$. Then $U(g)=D S(\pi(g)) D^{-1}$ defines a representation of $G$ on $L^{2}(S)$ such that each $U(g)$ restricts to an algebra automorphism of $L^{\infty}(S)$. It follows from Mackey's point realization theorem [7] that there is an ergodic action of $G$ on $S$ which induces $U(g)$. The representation $U$ restricted to $L^{2}(S) \ominus C$ is of course equivalent to $\Sigma_{k>1}^{\oplus} S^{k}(\pi)$. Each $S^{k}(\pi)$ is a subrepresentation of the tensor product of $\pi$ with itself $k$ times. As the tensor product of $\pi$ with itself is equivalent to a multiple of $\pi$ [3], it is clear that the proof of (i) will be complete once we have the following.

LEMMA 3.2. For the regular representation of any locally compact noncompact group, we have $\pi\left(g_{n}\right) \rightarrow 0$ weakly for any sequence $g_{n} \rightarrow \infty$. 
PRoof. Let $f, h \in L^{2}(G)$. For $\varepsilon>0$, there is a compact set $K$ such that $\int_{G-K}\left(|f|^{2}+|h|^{2}\right)<\varepsilon^{2}$. If $g \notin K^{-1} K$, then $K g^{-1} \cap K=\varnothing$. Thus

$$
\begin{aligned}
\left|\int(\pi(g) f) \bar{h}\right| & \leqslant \int_{K}|\pi(g) f||h|+\int_{G-K}|\pi(g) f||h| \\
& \leqslant\left(\int_{K}|\pi(g) f|^{2} \int_{K}|h|^{2}\right)^{1 / 2}+\left(\int_{G-K}|\pi(g) f|^{2} \int_{G-K}|h|^{2}\right)^{1 / 2} \\
& \leqslant\left(\int_{K^{-1}}|f|^{2}\right)^{1 / 2}\|h\|+\|f\| \varepsilon \leqslant(\|h\|+\|f\|) \varepsilon .
\end{aligned}
$$

Since $\varepsilon$ is arbitrary, the result follows.

To see (ii), we note that if $H \subset G$ is a closed subgroup, then the restriction of $\pi$ to $H$ is equivalent to a direct integral of representations all equivalent to the regular representation of $H$. If $U(H) f=f$ for some $f \neq 1$, then by taking a suitable projection, $f$ can be chosen to lie in a subspace on which $H$ acts like the regular representation. It follows that $H$ leaves some element in its regular representation invariant which implies that $H$ is compact.

COROLlary 3.3. For any ergodic G-space $Y$, other than translation on $G$ itself, $Y$ always has an ergodic extension with relatively invariant measure, and hence an extension such that $(X, Y)$ is an amenable pair.

Proof. If $Y \times S$ is not ergodic, it follows from [17, Proposition 3.1] that the $Y \times G$ cocycle res $(U)$ on $L^{2}(S) \ominus \mathbf{C}$ contains the identity. Then by Theorem 3.1 and [17, Corollary 3.3, Proposition 3.4], it follows that $Y=G / K$ where $K$ is a compact subgroup. But if $K$ does not reduce to the identity, $G$ is an extension of $Y$ with relatively invariant measure.

We remark that the basic observation used here that subgroups of an infinite dimensional unitary group may define natural ergodic actions (in contrast to the finite dimensional case) is due to I. E. Segal [11].

We now prove another existence theorem which shows that the collection of extensions with relatively invariant measure must be large enough to define most systems of imprimitivity based on $Y$, or equivalently, most unitary cocycles, that is, cocycles $\alpha: Y \times G \rightarrow U\left(H_{0}\right)$, where $U\left(H_{0}\right)$ is the unitary group of some Hilbert space $H_{0}$. Suppose $p: X \rightarrow Y$ is an extension with relatively invariant measure. Then, as described in [13], there is a naturally induced $Y \times G$ unitary cocycle $\alpha$. Namely we have for each $y, g$, a map $p^{-1}(y) \rightarrow p^{-1}(y g)$ which is measure preserving for almost all $y$. This induces a unitary $\alpha(y, g): H_{y g} \rightarrow H_{y}$, where $H_{y}=L^{2}\left(p^{-1}(y)\right)$. Almost all the $H_{y}$ are isomorphic by virtue of the ergodicity of $G$ on $Y$ and so we can suppose $\alpha(y, g) \in U\left(H_{0}\right)$ for some Hilbert space $H_{0}$. We want a condition under which a given cocycle $\beta$ on $Y \times G$ will be equivalent to a subcocycle of the 
naturally induced cocycle of some extension of $Y$. For any $\beta$, write $\beta \cong \gamma \oplus$ $\Sigma^{\oplus} d_{i} \beta_{i}$ where $\left\{\beta_{i}\right\}$ are a countable collection of finite dimensional unitary cocycles on $Y \times G, d_{i}$ are nonnegative integers, and $\gamma$ is a cocycle with no finite dimensional subcocycles.

THEOREM 3.4. If $d_{i} \leqslant \operatorname{dim} \beta_{i}$ for each $i$, then $\beta$ is equivalent to a subcocycle of the naturally induced cocycle of some ergodic extension $X$ of $Y$ with relatively invariant measure over $Y$.

Proof. We first show that $\gamma$ is a subcocycle of a naturally induced cocycle. We note first that $\gamma \oplus \bar{\gamma}$ is self-conjugate and so there is a conjugation on $H \oplus H$ which is $\gamma \oplus \bar{\gamma}$-invariant. It will suffice to show that $\gamma \oplus \bar{\gamma}$ is contained in a naturally induced cocycle, so we can actually assume that $H$ is the complexification of a real subspace $H_{0} \subset H$, with $H_{0} \gamma$-invariant. Let $S$ be as above, and define the cocycle $\tilde{\gamma}(y, g) \in U\left(L^{2}(S)\right)$ by $\tilde{\gamma}(y, g)=$ $D S(\gamma(y, g)) D^{-1}$. Let $X=Y \times S$ and define a unitary representation $\sigma$ of $G$ on $L^{2}(X) \cong L^{2}\left(Y, L^{2}(S)\right)$ by

$$
(\sigma(g) f)(y)=\tilde{\gamma}(y, g) f(y g) r(y, g)^{1 / 2} \quad \text { for } f \in L^{2}\left(Y, L^{2}(S)\right),
$$

where $r$ is the Radon-Nikodým cocycle of $Y \times G$. Since each $\tilde{\gamma}(y, g)$ is multiplicative on $L^{\infty}(S), \sigma(g)$ leaves the Boolean algebra $B(X)$ invariant, and hence by Mackey's point realization theorem there is a $G$-action on $X$ which induces the same Boolean action as $\sigma$. Since $\sigma(g)(1)=r(y, g)^{1 / 2}$, it follows that $\sigma$ is in fact the natural representation of $G$ associated to the action on $X$, and hence that $\tilde{\gamma}$ is the natural induced unitary $Y \times G$ cocycle on $L^{2}(S) \ominus \mathbf{C}$. Now $\tilde{\gamma} \cong \Sigma^{\oplus} S^{k}(\gamma)$, so we certainly have that $\gamma$ is a subcocycle of $\tilde{\gamma}$. It remains to show that $X$ is ergodic, and for this it suffices to see that $\tilde{\gamma}$ does not contain the identity cocycle [17, Proposition 3.1]. But since $\gamma$ has no finite dimensional subcocycles, the $k$-fold tensor product of $\gamma$ with itself does not contain the identity [13, Lemma 2.13] (recall $\gamma$ is self-conjugate). But since $S^{k}(\gamma)$ is a subcocycle of the $k$-fold tensor product, the result we are seeking is true for $\gamma$.

Now consider the representation $\gamma^{\prime}=\Sigma^{\oplus} d_{i} \beta_{i}, d_{i} \leqslant \operatorname{dim} \beta_{i}$. By [13, Theorem 6.4] there is an ergodic extension $Y \times S^{\prime}$ of $Y$ with relatively invariant measure such that the natural induced cocycle, say $\alpha$, contains $\gamma^{\prime}$. (Actually, [13, Theorem 6.4] is stated under the hypothesis that $Y$ has a finite invariant measure. However, the only point where this is used in the proof is in the proof of [13, Lemma 3.13], which by a different proof ([17, proof of Lemma 6.3], or [8]) is still valid for general $Y$.) With $S$ as above, we can now form the (fibered) product $Y \times S \times S^{\prime}$. The naturally induced cocycle of this extension will be $\tilde{\gamma} \otimes \alpha$. Since each of these contains the identity exactly once, $Y \times S \times S^{\prime}$ will be ergodic [13, Lemma 2.13], [17, Proposition 3.1], 
and it is clear that $\beta$ is a subcocycle of $\tilde{\gamma} \otimes \alpha$. This completes the proof.

When $Y$ is a point, we obtain the following result, which of course also follows directly by combining [11] with some classical results.

COROLLARY 3.15. If $\pi$ is a representation of $G$ sucn that each finite dimensional subrepresentation occurs with multiplicity less than or equal to its dimension, then $\pi$ is equivalent to a subrepresentation of the natural representation of some ergodic $G$-space with finite invariant measure.

4. The associated von Neumann algebras and conditional invariant means. In this section we turn to an examination of the connections between amenability of a pair, the existence of conditional invariant means, and relationships between various von Neumann algebras associated to a pair of groups or ergodic actions of countable discrete groups. In [16, generalizing known results for groups, we characterize amenable ergodic actions of countable discrete groups both in terms of the von Neumann algebra associated to it by the Murray-von Neumann construction, and the existence of an analogue of a $G$-invariant mean. The characterization in terms of von Neumann algebras concerns the existence of a norm one projection from the algebra of all bounded operators to the von Neumann algebra in question. This property, and properties related to it, have been studied for some time by a number of authors, culminating in the work of A. Connes [1]. Here, we shall characterize amenable pairs in terms of the existence of a norm one projection from one algebra to another, and show that amenability of the pair is equivalent to the existence of a conditional invariant mean.

We begin with a characterization in terms of von Neumann algebras of amenability for a pair of groups. Let $G$ be a locally compact second countable group and $H \subset G$ a closed subgroup. Let $U(g)$ be the right regular representation of $G$ on $L^{2}(G), V(g)$ the left regular representation, $R(G)=$ $\{U(g)\}^{\prime \prime}, R(H)=\{U(h)\}^{\prime \prime}$, and define $L(G)$ and $L(H)$ analogously. Although we do not need this fact, we note that $R(H)$ is isomorphic to the von Neumann algebra generated by the right regular representation of $H$ on $L^{2}(H)$.

THEOREM 4.1. If $(H, G)$ is an amenable pair, then there is a norm one projection of $L(H)^{\prime}$ onto $L(G)^{\prime}=R(G)$. The converse is true if $G$ is countable and discrete.

Proof. Suppose first that $(H, G)$ is an amenable pair, that is, has the conditional fixed point property of Eymard. For $T \in L(H)^{\prime}$, let $C(T)$ be the weakly closed convex hull of $\left\{V(g) T V(g)^{-1} \mid g \in G\right\}$. Then there is a natural affine action of $G$ on $C(T)$. Since $T \in L(H)^{\prime}, T$ is a fixed point for the action of $H$ on $C(T)$. By the conditional fixed point property, there is a 
fixed point in $C(T)$ for $G$, that is, an element of $C(T) \cap L(G)^{\prime}$. Arguing as in the proof of $[9,4.4 .15]$, we obtain the norm one projection.

Now suppose that $G$ is countable and discrete and that $P: L(H)^{\prime} \rightarrow L(G)^{\prime}$ is a norm one projection. Let $G / H$ be the cosets of the form $\{H g\}$. Then we have a natural identification $L^{\infty}(G / H) \subset L^{\infty}(G)$, and a representation $M$ of $L^{\infty}(G)$ as multiplication operators on $L^{2}(G)$. Clearly $M\left(L^{\infty}(G / H)\right) \subset$ $L(H)^{\prime}$. For $f \in L^{\infty}(G / H)$, let $m(f)=\langle P(M(f)) x \mid x\rangle$ where $x$ is the characteristic function of the identity element in $G$. Let $f \cdot g$ be the right translation of $f$. Then

$$
\begin{aligned}
m(f \cdot g) & =\left\langle P\left(U(g)^{-1} M(f) U(g)\right) x \mid x\right\rangle \\
& =\left\langle U(g)^{-1} P(M(f)) U(g) x \mid x\right\rangle \quad \text { since } U(g) \in L(G)^{\prime} \\
& =\left\langle U(g)^{-1} V(g)^{-1} P(M(f)) V(g) U(g) x \mid x\right\rangle \text { since } V(g) \in L(G) \\
& =m(f) \text { since } U(g) V(g) x=x .
\end{aligned}
$$

Clearly $m(1)=1$ and $\|m\|=1$. Thus $m$ is a $G$-invariant mean on $L^{\infty}(G / H)$ which implies that $(H, G)$ is an amenable pair.

We now turn to the analogous result for amenable pairs of ergodic actions of countable discrete groups. We begin with some general results we will need concerning extensions and Borel fields of compact convex sets. Let $G$ be a countable discrete group, $(X, m)$ and $(Y, \nu)$ ergodic $G$-spaces, and $p: X \rightarrow Y$ a Borel $G$-map. By virtue of the ergodicity of $G$ on $Y, X$ will be isomorphic (modulo null sets) as an extension of $Y$ to a product $(X, m)=(Y \times I$, $\left.\nu \times \mu_{0}\right)$, where $I$ is the unit interval, $\mu_{0}$ is a Borel measure on $I$, and $p: X \rightarrow Y$ is just given by projection on the first factor. Let $E$ be a separable Banach space. We recall that for a standard Borel space $(S, \mu)$, we have $L^{1}(S, E)^{*} \cong$ $L^{\infty}\left(S, E^{*}\right)$. By the $\sigma$-topology on $L^{\infty}\left(S, E^{*}\right)$, we mean the $\sigma\left(L^{\infty}\left(S, E^{*}\right)\right.$, $\left.L^{1}(S, E)\right)$ topology, and similarly, by the $\sigma$-topology on $E^{*}$, we mean the $\sigma\left(E^{*}, E\right)$ topology. By $L^{\infty}\left(S, E_{1}^{*}\right)$ we mean the unit ball in $L^{\infty}\left(S, E^{*}\right)$, which is compact and metrizable in the $\sigma$-topology. We shall examine some relations between Borel fields of compact convex sets on $X=Y \times I$ and those on $Y$. The proof of the following is routine, and we omit it.

LEMMA 4.1. The map $L^{\infty}\left(Y, E_{1}^{*}\right) \rightarrow L^{\infty}\left(X, E_{1}^{*}\right)$ defined by composition with $p$ is $\sigma$-continuous (and of course norm continuous).

Now let $f: I \rightarrow E_{1}^{*}$ be a Borel function, where $E_{1}^{*}$ has the $\sigma$-topology, and let ess $(f)$ be the essential range of $f$ with respect to the measure $\mu_{0}$ on $I$. For any probability measure $\mu$ on $I$, we have $f_{*}(\mu) \in M\left(E_{1}^{*}\right)$, the probability measures on $E_{1}^{*}$. Let $b: M\left(E_{1}^{*}\right) \rightarrow E_{1}^{*}$ be the barycenter map. Then $b$ is continuous where $M\left(E_{1}^{*}\right)$ has the $\sigma\left(M\left(E_{1}^{*}\right), C\left(E_{1}^{*}\right)\right)$ topology (which we also 
call the $\sigma$-topology on $\left.M\left(E_{1}^{*}\right)\right)$. For any set $A \subset E_{1}^{*}$, let $\overline{\operatorname{co}}(A)$ be the closed convex hull of $A$.

LEMMA 4.2. There exists a countable collection of probability measures $\mu_{n}$ on $I$, absolutely continuous with respect to $\mu_{0}$, such that for every Borel function f: $I \rightarrow E_{1}^{*}$, we have

$$
\overline{\operatorname{co}}(\operatorname{ess}(f))=\left\{\overline{b\left(f_{*}\left(\mu_{n}\right)\right)}\right\} .
$$

This set $\left\{\mu_{n}\right\}$ is independent of $E$.

PROof. We note first that for any $\mu \ll \mu_{0}, f_{*}(\mu)$ is supported on the closed set ess $(f)$, and hence $b\left(f_{*}(\mu)\right) \in \operatorname{co}(\operatorname{ess}(f))$. Now let $\mathscr{F}$ be a countable generating field for the Borel structure on $I$. For each Borel $A \subset I$ with $\mu_{0}(A)>0$, let $\mu_{A}$ be the induced probability measure on $A$, that is $\mu_{A}(B)=$ $\mu_{0}(A \cap B) / \mu_{0}(A)$, and $\left\{\mu_{n}\right\}$ the countable set of measures on $I$ obtained by taking finite rational convex combinations of $\left\{\mu_{A} \mid A \in \mathscr{F}, \mu_{0}(A)>0\right\}$. Since $f_{*}$ and $b$ are affine functions, we conclude that $\left\{\overline{b\left(f_{*}\left(\mu_{n}\right)\right)}\right\}$ is a convex set contained in $\overline{c o}(\operatorname{ess}(f))$. Thus, it suffices to see that if $x \in \operatorname{ess}(f)$, then $x \in\left\{\overline{b\left(f_{*}\left(\mu_{n}\right)\right)}\right\}$. Choose a compact convex neighborhood of $x$ in $E_{1}^{*}$, say $W$. Since $x \in \operatorname{ess}(f), \mu_{0}\left(f^{-1} W\right)>0$. For each $n$, choose $A_{n} \in \mathcal{F}$ such that $\mu_{0}\left(A_{n} \Delta f^{-1}(W)\right)<1 / n$. Since $M\left(E_{1}^{*}\right)$ is compact, we can suppose, by passing to a subsequence if necessary, that $f_{*}\left(\mu_{A_{n}}\right) \sigma$-converges to a probability measure $\nu$ on $E_{1}^{*}$. Then $b\left(f_{*}\left(\mu_{A_{n}}\right)\right) \rightarrow b(\nu)$, and since there is a countable neighborhood basis of $x$ consisting of compact convex sets, it suffices to show $b(\nu) \in W$. If not, there is an element $y \in E$ such that $\langle b(\nu), y\rangle>0$ and $\langle w, y\rangle\left\langle 0\right.$ for all $w \in W$. But then $\left\langle b\left(f_{*}\left(\mu_{A_{n}}\right)\right), y\right\rangle>0$ for all sufficiently large $n$, that is,

$$
\int\langle f(t), y\rangle \chi_{A_{n}} d \mu_{0}(t) / \mu_{0}\left(A_{n}\right)>0 .
$$

Since $y$ is bounded on $E_{1}^{*}$, as $n \rightarrow \infty$ this becomes

$$
\int\langle f(t), y\rangle \chi_{f^{-1} W} d \mu_{0}(t) / \mu_{0}\left(f^{-1} W\right)>0 .
$$

But this contradicts the fact that $\langle w, y\rangle\langle 0$ for all $w$, proving the lemma.

LEMMA 4.3. For each probability measure $\mu \ll \mu_{0}$, the map $L^{\infty}\left(I, E_{1}^{*}\right) \rightarrow E_{1}^{*}$ defined by $f \rightarrow b\left(f_{*}(\mu)\right)$ is continuous, where both spaces have the $\sigma$-topology.

Proof. If $x \in E$ and $f_{n} \rightarrow f$ in $L^{\infty}\left(I, E_{1}^{*}\right)$, we must show that $\left\langle b\left(\left(f_{n}\right)_{*}(\mu)\right), x\right\rangle \rightarrow\left\langle b\left(f_{*}(\mu)\right), x\right\rangle$. The left side is

$$
\int\left\langle f_{n}(t), x\right\rangle d \mu=\int\left\langle f_{n}(t),\left(d \mu / d \mu_{0}\right)(t) x\right\rangle d \mu_{0} .
$$

Now $t \rightarrow\left(d \mu / d \mu_{0}\right)(t) x$ is in $L^{1}\left(I, \mu_{0}, E\right)$. Thus as $n \rightarrow \infty$, we obtain by the 
$\sigma$-convergence of $f_{n}$ to $f$,

$$
\int\left\langle f(t),\left(d \mu / d \mu_{0}\right)(t) x\right\rangle d \mu_{0}=\left\langle b\left(f_{*}(\mu)\right), x\right\rangle .
$$

Now suppose that $f: Y \times I \rightarrow E_{1}^{*}$ is Borel. For each $y$, we have $f_{y}: I \rightarrow E_{1}^{*}$ defined by $f_{y}(t)=f(y, t)$.

LEMMA 4.4. If $f: Y \times I \rightarrow E_{1}^{*}$ is Borel, and $\mu \ll \mu_{0}$ is a probability measure on I, define $\Phi_{\mu}(f)(y)=b\left(\left(f_{y}\right)_{*}(\mu)\right)$. Then $\Phi_{\mu}(f): Y \rightarrow E_{1}^{*}$ is Borel.

Proof. The map $Y \rightarrow L^{\infty}\left(I, E_{1}^{*}\right)$ defined by $y \rightarrow f_{y}$ is Borel (by Fubini's theorem) and the result now follows from Lemma 4.3.

Corollary 4.5. If $f: Y \times I \rightarrow E_{1}^{*}$ is Borel, then $y \rightarrow \overline{\operatorname{co}}\left(\operatorname{ess}\left(f_{y}\right)\right)$ is a Borel field of compact convex subsets of $E_{1}^{*}$.

Proof. This follows from Lemma 4.2, Lemma 4.4, and [14, Lemma 1.7].

We shall make important use of the following lemma, whose proof is straightforward.

LEMMA 4.6. Suppose $\alpha: Y \times G \rightarrow \operatorname{Iso}(E)$ is a cocycle. Let $\beta(x, g)=$ $\alpha(p(x), g)$. If $f: X \rightarrow E_{1}^{*}$ is a $\beta$-invariant section, then $y \rightarrow \overline{\operatorname{co}}\left(\operatorname{ess}\left(f_{y}\right)\right)$ is an $\alpha$-invariant Borel field of compact convex sets.

We now present the amenability criteria for a pair of ergodic $G$-spaces. As above, we let $U(g)$ and $V(g)$ denote the right and left regular representations of $G$ respectively, acting on $L^{2}(G)$. The following operators are defined on $L^{2}(X \times G)$. For $g \in G$, let

$$
(\tilde{U}(g) f)(x, h)=f(x g, h g) r(x, g)^{1 / 2}, \quad(\tilde{V}(g) f)(x, h)=f\left(x, g^{-1} h\right) ;
$$

and for $s \in L^{\infty}(X)$, define

$$
\left(M_{s} f\right)(x, h)=s(x) f(x, h), \quad\left(N_{s} f\right)(x, h)=s(x h) f(x, h) .
$$

Let $R$ be the von Neumann algebra generated by $\left\{\tilde{U}(g), M_{s} \mid g \in G, s \in\right.$ $\left.L^{\infty}(X)\right\}, L$ the von Neumann algebra generated by $\left\{\tilde{V}(g), N_{s} \mid g \in G\right.$, $\left.s \in L^{\infty}(X)\right\}$, and $L_{Y}$ the von Neumann algebra generated by $\left\{\tilde{V}(g), N_{s} \mid g \in\right.$ $G, s=u \circ p$ where $\left.u \in L^{\infty}(Y)\right\}$. We then have the main theorem of this section, which includes a converse to Proposition 2.9 for suitable $G$.

THEOREM 4.7. Let $G$ be a countable discrete group, $p: X \rightarrow Y$ an extension of ergodic $G$-spaces. Then the following are equivalent:

(i) $(X, Y)$ is an amenable pair.

(ii) There is a norm one projection from $L$ onto $L_{Y}$.

(iii) $X$ has a $G$-invariant conditional mean over $Y$.

When $Y$ is a point, we obtain in particular the following corollary. 
COROllaRy 4.8. If $G$ is a countable discrete group and $X$ is an ergodic $G$-space, then $(X, G)$ is an amenable pair if and only if there is a G-invariant mean on $L^{\infty}(X)$.

We note that for arbitrary $G$, Eymard has shown this is true for transitive $X$ [4].

Before beginning the proof, we collect some facts we will need. We can write $L^{2}(X \times G)$ in a natural way as a direct integral, $L^{2}(X \times G)=$ $\int_{X}^{\oplus} L^{2}(G)$, where the isomorphism takes $f(x, g) \in L^{2}(X \times G)$ into the field $x \rightarrow f_{x}$, where $f_{x} \in L^{2}(G)$ is given by $f_{x}(g)=f(x, g)$. Under this isomorphism $\tilde{V}(g)$ corresponds to the direct integral of the constant field of operators $\tilde{V}(g)=\int_{X}^{\oplus} V(g)$. Each operator $N_{s}, s \in L^{\infty}(X)$, is clearly also decomposable with respect to this direct integral decomposition. Thus, letting $D$ be the algebra of decomposable operators, we have $L \subset D$. We note that $D$ can be naturally identified with $L^{\infty}\left(X, B\left(L^{2}(G)\right)\right)$, where $B\left(L^{2}(G)\right)$ is the algebra of bounded operators on $L^{2}(G)$. Let $T\left(L^{2}(G)\right.$ ) be the separable Banach space of trace class operators on $L^{2}(G)$ with the tracial norm. We have $T\left(L^{2}(G)\right)^{*}=B\left(L^{2}(G)\right)$, and $L^{1}\left(X, T\left(L^{2}(G)\right)\right)^{*} \cong L^{\infty}\left(X, B\left(L^{2}(G)\right)\right)$. On bounded sets the weak operator topology on $D$ corresponds to the $\sigma$-topology on $L^{\infty}\left(X, B\left(L^{2}(G)\right)\right)$. We can identify $L^{\infty}\left(Y, B\left(L^{2}(G)\right)\right)$ via an imbedding $J$ as a subalgebra of $D$. Namely if $T \in L^{\infty}\left(Y, B\left(L^{2}(G)\right)\right)$, let $(J(T))(x)=T(p(x))$. Then $J$ will be continuous in norm and in the weak operator topology on bounded sets. Let us denote the image of $J$ by $D_{Y} \subset D$. Then it is clear that $\tilde{V}(g)$ and $N_{s}, s \in L^{\infty}(Y)$, are contained in the von Neumann algebra $D_{Y}$, and hence $L_{Y} \subset D_{Y}$. We shall denote by $\tilde{V}^{Y}(g)$ and $N_{s}^{Y}$ the elements in $L^{\infty}\left(Y, B\left(L^{2}(G)\right)\right)$ corresponding to $\tilde{V}(g)$ and $N_{s}$ respectively. These operators act on the Hilbert space $L^{2}\left(Y, L^{2}(G)\right)$. We can also define operators $\tilde{U}^{Y}(g)$ and $M_{s}^{Y}, g \in G, s \in L^{\infty}(Y)$, in an entirely analogous manner. We note that $L_{Y}=J\left(\left\{V^{Y}(g), N_{s}^{Y}\right\}^{\prime \prime}\right)$, and recall the classical result that $R^{\prime}=L$ [18, pp. 137-138] (and similarly for the algebras defined on $\left.L^{2}\left(Y, L^{2}(G)\right)\right)$. We now turn to the proof of the theorem.

Proof (of Theorem 4.7), (i) $\Rightarrow$ (ii). Let $D$ be as above, and $B(D)$ the bounded operators on $D$. Then $B(D)=\left(D \otimes_{\max } D_{*}\right)^{*}$, and we call the $\sigma\left(B(D), D \otimes_{\max } D_{*}\right)$ topology the $\sigma$-topology. Let $T=\int^{\oplus} T(x) \in D$. Then for $s \in L^{\infty}(Y)$, define $\Phi_{s} \in B(D)$ by $\Phi_{s} T=M_{s} \circ p$, and for a probability measure $\mu$ on $I, \mu \ll \mu_{0}$, define $\Phi_{\mu}(T)(x)=b\left(\left(T_{p(x)}\right)_{*}(\mu)\right)$. We note that $\Phi_{\mu}(T) \in D$ by Lemma 4.4. Let $F \subset B(D)$ be the set of all finite sums $\Sigma \Phi_{s_{i}} \Phi_{\mu_{i}}$ where $\mu_{i} \in\left\{\mu_{n}\right\}$ are chosen as in Lemma 4.2, and $s_{i} \in L^{\infty}(Y)$ with $s_{i} \geqslant 0$ and $\Sigma s_{i}=1$. We easily see that $F$ is convex and that for $\Phi \in F,\|\Phi\| \leqslant 1$. Let $\bar{F}$ be the $\sigma$-closure of $F$, so that $\bar{F}$ is a compact convex set. We recall that if $T \in D$ then the map $\bar{F} \rightarrow D$ given by $\Phi \rightarrow \Phi(T)$ is continuous where $\bar{F}$ and 
$D$ have the $\sigma$-topologies. The essence of the proof of (i) implies (ii) is the following.

LEMMA. For each finite set $A_{1}, \ldots, A_{n} \in L$, there is a $\Phi \in \bar{F}$ such that $\Phi\left(A_{i}\right) \in L_{Y}$ for each $i$.

Proof. Let $E$ be the separable Banach space $T\left(L^{2}(G)\right) \oplus \cdots \oplus$ $T\left(L^{2}(G)\right)$, the direct sum taken $n$ times. Then $E^{*}=B\left(L^{2}(G)\right) \oplus \cdots \oplus$ $B\left(L^{2}(G)\right)$. Let $\alpha: Y \times G \rightarrow \operatorname{Iso}\left(T\left(L^{2}(G)\right)\right)$ be defined by $\alpha(y, g) T=$ $U(g) T U(g)^{-1}$. Then the adjoint cocycle $\alpha^{*}: Y \times G \rightarrow \operatorname{Iso}\left(B\left(L^{2}(G)\right)\right)$ is given by the same formula. Let $\tilde{\alpha}: Y \times G \rightarrow \operatorname{Iso}(E)$ be $\tilde{\alpha}=\alpha \oplus \cdots \oplus \alpha$, so that $\tilde{\alpha}^{*}=\alpha^{*} \oplus \cdots \oplus \alpha^{*}$. We define $\beta$ and $\tilde{\beta}$ to be the restrictions of $\alpha$ and $\tilde{\alpha}$ to $X \times G$, that is, $\beta(x, g)=\alpha(p(x), g), \tilde{\beta}(x, g)=\tilde{\alpha}(p(x), g)$. Let $A \in$ $L^{\infty}\left(X, E_{1}^{*}\right)$ be defined by $A(x)=\left(A_{1}\right)(x) \oplus \cdots \oplus\left(A_{n}\right)(x)$. Since each $A_{i}$ commutes with $\tilde{U}(g)$, we have $U(g)^{-1}\left(A_{i}\right)(x) U(g)=A_{i}(x g)$ a.e., which implies that $A(x)$ is a $\tilde{\beta}$-invariant section. Let $C_{y}=\overline{\operatorname{co}}\left(\operatorname{ess}\left(A_{y}\right)\right) \subset E_{1}^{*}$. By Lemma 4.5, $C_{y}$ is an $\tilde{\alpha}$-invariant field of compact convex sets, and clearly $A(x) \in$ $C_{p(x)}$ a.e. Thus, by the definition of amenable pair, there is an $\tilde{\alpha}$-invariant section, say $W(y) \in C_{y}$. From Lemma 4.2 and [16, Lemma 2.2], we conclude that $W \in L^{\infty}\left(Y, E_{1}^{*}\right)$ is the $\sigma$-limit of elements $W_{k}$ where each $W_{k}$ is a finite sum,

$$
W_{k}(y)=\sum f_{k i}(y) b\left(\left(A_{y}\right)_{*}\left(\mu_{k i}\right)\right)
$$

with $f_{k i} \geqslant 0$ and $\Sigma_{i} f_{k i}=1$. But this can be written $W_{k}(y)=W_{k 1}(y)$ $\oplus \cdots \oplus W_{k n}(y)$ where

$$
W_{k j}(y)=\sum_{i} f_{k i}(y) b\left(\left(\left(A_{j}\right)_{y}\right)_{*}\left(\mu_{k i}\right)\right)
$$

We have $W(y)=W_{1}(y) \oplus \cdots \oplus W_{n}(y)$, where $W_{j}=\sigma-\lim W_{k j}$ as $k \rightarrow \infty$. Since $W$ is $\tilde{\alpha}$-invariant, it follows that $U(g)^{-1} W_{j}(y) U(g)=W_{j}(y g)$ a.e., or equivalently, $W_{j}$ commutes with $\tilde{U}^{Y}(g)$. As it clearly commutes with $M_{s}^{Y}$ for all $s \in L^{\infty}(Y)$, it follows that $W_{j}$ is in the von Neumann algebra generated by $\tilde{V}^{Y}(g), N_{s}^{Y}$, and hence that $T_{j}=J\left(W_{j}\right) \in L^{\infty}\left(X, B\left(L^{2}(G)\right)\right)$ is actually contained in $L_{Y}$. Let $\Phi^{k} \in F$ be defined by $\Phi^{k}=\Sigma \Phi_{f_{k i}} \Phi_{\mu_{k i}}$. Since $W_{j}=\sigma$ $\lim W_{k j}$, it follows that $T_{j}=\sigma$-lim $\Phi^{k}\left(A_{j}\right)$. By the compactness of $\bar{F},\left\{\Phi^{k}\right\}$ has a $\sigma$-accumulation point $\Phi \in \vec{F}$. It is clear that $\Phi\left(A_{j}\right)=T_{j}$, and since we have already established that $T_{j} \in L_{Y}$, the proof of the lemma is complete.

We now complete the proof of (i) $\Rightarrow$ (ii). For each $A \in L$, let $F_{A}=\{\Phi \in$ $\left.\bar{F} \mid \Phi(A) \in L_{Y}\right\}$. For each $A, \Phi \rightarrow \Phi(A)$ is $\sigma$-continuous into $D$, and since $L_{Y} \subset D$ is $\sigma$-closed, $F_{A}$ is compact. The lemma implies that the intersection of any finite number of the $F_{A}$ is nonempty, and so by the finite intersection property, there is $\Phi \in \bar{F}$ such that $\Phi(L) \subset L_{Y}$. Since $L_{Y} \subset D_{Y}$, it is clear from the form of an element in $F$ that any member of $F$ is the identity on $L_{Y}$, 
and hence the same is true for elements of $\bar{F}$. Hence, $\Phi$ is the required norm one projection.

Proof of (ii) $\Rightarrow$ (iii). Let $P: L \rightarrow L_{Y}$ be a norm one projection. For $f \in L^{\infty}(X)$, we have the operator $N_{f} \in L$. Let $a \in L^{2}(G)$ be the function which is one on the identity and vanishes elsewhere. Then $P\left(N_{f}\right) \in L_{Y} \subset$ $J\left(L^{\infty}\left(Y, B\left(L^{2}(G)\right)\right)\right)$. Define $\sigma(f)(y)=\left\langle J^{-1} P\left(N_{f}\right)(y) a \mid a\right\rangle$. Since $\tilde{V}(g) \in$ $L_{Y}$, we have $P\left(\tilde{V}(g)^{-1} N_{f} \tilde{V}(g)\right)=\tilde{V}(g)^{-1} P\left(N_{f}\right) \tilde{V}(g)$ [12]. Since $P\left(N_{f}\right)$ commutes with $\tilde{U}(g)$, we also have

$$
U(g)^{-1}\left(J^{-1} P\left(N_{f}\right)(y)\right) U(g)=J^{-1} P\left(N_{f}\right)(y g)
$$

for each $g$ and almost all $y$. Thus for almost all $y$,

$$
\begin{aligned}
\sigma(f \cdot g)(y) & =\left\langle J^{-1} P\left(N_{f \cdot g}\right)(y) a \mid a\right\rangle \\
& =\left\langle J^{-1} P\left(\tilde{V}(g)^{-1} N_{f} \tilde{V}(g)\right)(y) a \mid a\right\rangle \\
& =\left\langle V(g)^{-1}\left(J^{-1} P\left(N_{f}\right)(y)\right) V(g) a \mid a\right\rangle \\
& =\left\langle V(g)^{-1} U(g)^{-1}\left(J^{-1} P\left(N_{f}\right)(y g)\right) U(g) V(g) a\right| a^{\prime}, \\
& =\left\langle J^{-1} P\left(N_{f}\right)(y g) a \mid a\right\rangle \\
& =\sigma(f)(y g)=(\sigma(f) \cdot g)(y) .
\end{aligned}
$$

This shows that $\sigma$ is a $G$-map. Furthermore, if $f \in L^{\infty}(Y)$, then $J^{-1} P\left(N_{f}\right)=$ $N_{f}^{Y}$, and $\left(N_{f}^{Y}(y)\right)(a)(g)=f(y g) a(g)$. Hence $\left\langle N_{f}^{Y} a \mid a\right\rangle=f(y)$, showing that $\sigma(f)=f$ for $f \in L^{\infty}(Y)$. Hence $\sigma$ is a norm one projection $\sigma: L^{\infty}(X) \rightarrow$ $L^{\infty}(Y)$, completing the proof.

(iii) $\Rightarrow$ (i). This is just Proposition 2.9.

\section{REFERENCES}

1 A. Connes, Classification of injective factors, Ann. of Math. (2) 104 (1976), 73-115.

2. R. E. Edwards, Functional analysis, Holt, New York, 1965.

3. J. Ernest, Hopf-von Neumann algebras, Functional Analysis, Editor, B. R. Gelbaum, Academic Press, London, 1967.

4. P. Eymard, Moyennes invariantes et représentations unitaires, Lecture Notes in Math., no. 300, Springer-Verlag, New York, 1972.

5. S. Glasner, Relatively invariant measures, Pacific J. Math. 58 (1975), 393-400.

6. H.-H. Kuo, Gaussian measures in Banach spaces, Lecture Notes in Math., no. 463, Springer-Verlag, New York, 1975.

7. G. W. Mackey, Point realization of transformation groups, Illinois J. Math. 6 (1962), 327-335.

8. A. Ramsay, Non-transitive quasi-orbits in Mackey's analysis of group extensions, Acta Math. 137 (1976), $17-48$.

9. S. Sakai, $C^{*}$-algebras and $W^{*}$-algebras, Springer-Verlag, New York, 1971.

10. I. E. Segal, Tensor algebras over Hilbert spaces. I, Trans. Amer. Math. Soc. 81 (1956), 106-134. 
11. __ Ergodic subgroups of the orthogonal group of a real Hilbert space, Ann. of Math. (2) 66 (1957), 297-303.

12. J. Tomiyama, On the projection of norm one in $W^{*}$-algebras, Proc. Japan Acad. 33 (1957), 608-612.

13. R. J. Zimmer, Extensions of ergodic group actions, Illinois J. Math. 81 (1976), 373-409.

14. Amenable ergodic group actions and an application to Poisson boundaries of random walks, J. Functional Analysis 27 (1978), 350-372.

15. On the von Neumann algebra of an ergodic group action, Proc. Amer. Math. Soc. 66 (1977), 289-293.

16. , Hyperfinite factors and amenable ergodic actions, Invent. Math. 41 (1977), 23-31.

17. _ Orbit spaces of unitary representations, ergodic theory, and simple Lie groups, Ann. of Math. (2) 106 (1977), 573-588.

18. J. Dixmier, Les algèbres d'opérateur dans l'espace Hilbertien, Gauthier-Villars, Paris, 1969.

Department of Mathematics, University of Chicago, Chicago, Ilunnois 60637 